Instituto Internacional de Investigación y Desarrollo Tecnológico Educativo INDTEC, C.A.

DOI: $h$ ttps://doi.org/10.29394/Scientific.issn.2542-2987.2020.5.15.5.106-128

OAI-PMH: http://www.indteca.com/ojs/index.php/Revista Scientific/oai

Artículo Original / Original Article

\title{
Rol del docente investigador desde su práctica social
}

\author{
Autoras: Eddymar María Flores Nessi \\ Universidad Politécnica Territorial del Estado Zulia, UPTZ \\ eddymarf.nessi@gmail.com \\ Zulia, Venezuela \\ https://orcid.org/0000-0003-1786-2283 \\ Alba Carolina Loaiza Falcón \\ Universidad Politécnica Territorial del Estado Zulia, UPTZ \\ albaloaiza.104@gmail.com \\ Zulia, Venezuela \\ https://orcid.org/0000-0002-5563-4911 \\ Gisemar Ninoska Rojas de Ricardo \\ Universidad Nacional Experimental "Rafael María Baralt", UNERMB \\ gisemarninoska@gmail.com \\ Zulia, Venezuela \\ https://orcid.org/0000-0002-1723-3340
}

\section{Resumen}

En este artículo, se aborda desde el punto de vista teórico el rol del docente investigador de la unidad curricular proyectos, considerada unidad neurálgica de los actuales programas universitarios, donde se articulan diferentes saberes y acciones para la ejecución de investigaciones que conduzcan a la transformación social. Ante el proceso de transformación universitaria en Venezuela, se hace necesario potenciar la función del docente como investigador, tutor, asesor, facilitador y mediador de aprendizajes que contribuya a la formación integral del estudiante con una visión crítica para la solución de los problemas del entorno. Teóricamente se fundamentó en los postulados de Hernández (2009); Saby (2012); Castro (2014); y Morillo (2009); entre otros. La metodología utilizada fue la revisión bibliográfica consultándose trabajos de investigaciones y artículos de revistas especializadas. Para ello se plantean las diferentes acciones y estrategias para mejorar el desempeño del docente durante la elaboración de proyectos de investigación, a través del desarrollo de competencias que abarcan la integralidad del ser humano. Finalmente se presentan reflexiones en torno al rol del docente ante la práctica de investigación científica y su relación con el proceso de enseñanzaaprendizaje en el contexto de la educación universitaria.

Palabras clave: docente; proyecto de investigación; transformación social.

Cómo citar este artículo:

Flores, E., Loaiza, A., \& Rojas de Ricardo, G. (2020). Rol del docente investigador desde su práctica social. Revista Scientific, 5(15), 106-128, e-ISSN: 2542-2987. Recuperado de: https://doi.org/10.29394/Scientific.issn.2542-2987.2020.5.15.5.106-128

Fecha de Recepción: 28-06-2019
Fecha de Aceptación: 01-10-2019
Fecha de Publicación: 05-02-2020 


\title{
Role of the research teacher from his social practice
}

\begin{abstract}
In this article, from the theoretical point of view the role of the research teacher of the curricular unit projects, considered as the nerve unit of the current university programs, where different knowledge and actions for the execution of research that lead to social transformation are articulated. Given the process of university transformation in Venezuela, it is necessary to strengthen the role of the teacher as a researcher, tutor, advisor, facilitator and mediator of learning that contributes to the integral formation of the student with a critical vision for the solution of environmental problems. Theoretically it was based on the postulates of Hernández (2009); Saby (2012); Castro (2014); and Morillo (2009); among others. The methodology used was the literature review consulting research papers and articles of specialized journals. For this, the different actions and strategies to improve teacher performance during the development of research projects are proposed, through the development of competences that encompass the integrality of the human being. Finally, reflections are presented regarding the role of the teacher in the practice of scientific research and its relationship with the teaching-learning process in the context of university education.
\end{abstract}

Keywords: teacher; research project; social change.

How to cite this article:
Flores, E., Loaiza, A., \& Rojas de Ricardo, G. (2020). Rol del docente investigador desde su práctica
social. Revista Scientific, $\quad 5(15), \quad 106-128, \quad$ e-ISSN: $2542-2987 . \quad$ Recovered from:
https://doi.org/10.29394/Scientific.issn.2542-2987.2020.5.15.5.106-128

Date Received: 28-06-2019
Date Acceptance:

01-10-2019
Date Publication: 05-02-2020 


\section{Introducción}

En Venezuela en el año 2008 y a través del proyecto político de País en conjunto con el Ministerio del Poder Popular para la Educación Universitaria, anteriormente llamado Ministerio de Educación Superior para ese entonces, según Gaceta Oficial N. ${ }^{\circ} 38.930$ de fecha 13 de mayo del año 2008, se crea los Programas Nacionales de Formación (PNF), con el fin de proponer modificaciones a los pensum de estudios de carreras universitarias en función de darles respuestas a las exigencias de la sociedad.

Los PNF son asumidos como entornos de aprendizaje colectivo a través de la Unidad Curricular (UC) Proyectos, donde los estudiantes en conjunto con docentes y asesores interactúan constantemente con las comunidades para llevar a cabo trabajos de investigación, en los cuales se articulan distintos saberes para satisfacer las demandas del sector y finalmente validar los procesos formativos y de investigación.

A partir de la transformación y modernización de los Institutos Universitarios de Tecnología (IUT) y Colegios Universitarios (CU) en Universidades Politécnicas Territoriales (UPT) incorporando en estas los PNF, se consagra la nueva misión de la educación universitaria orientada a promover el desarrollo científico y tecnológico mediante prácticas sociales, a través de la ejecución de proyectos acordes a las necesidades del entorno, y con el fin de promover productos de investigación que se traduzcan en bien o servicio e incorporando la dimensión productiva en las comunidades.

Dentro de este contexto, estas nuevas universidades a través de los PNF ofrecen carreras cortas y largas a nivel de pregrado, con carácter terminal de Técnico Superior Universitario (TSU) en un periodo de dos (02) años, con posibilidad de prosecución de estudios con carácter de Ingeniería o Licenciatura en un periodo de dos (02) años subsiguientes, orientados a darles respuestas a sectores estratégicos de la economía nacional como: agroalimentación, farmacia, industrias básicas, hidrocarburos, petroquímica, 
minería, construcción, telecomunicaciones, servicios básicos, entre otros.

Por su parte, desde el inicio hasta el final de la formación académica, el estudiante en compañía del docente de proyectos, se inserta en las comunidades a recabar información requerida para realizar un diagnóstico de necesidades o potencialidades, que lo llevan a formalizar un proyecto que ejecutarán durante los trayectos de formación. Dentro de estos espacios se comparten con las comunidades los diferentes aprendizajes adquiridos de las demás unidades curriculares que tributan a proyectos, permitiendo la integración social y dando respuestas a las necesidades del sector.

En este orden de ideas, Rodríguez (2016), plantea que los docentes que administran unidades curriculares de Proyectos: "tienen responsabilidades administrativas y gerenciales enmarcadas hacia la descentralización, la aplicación del Proceso de Aprendizaje Socio Integrador Productivo" (pág. 28). Asimismo, el docente debe desempeñase como gerente de aula con vocación de servicio, calidad humana y carisma para inducir a los estudiantes a aprender-aprender y buscar la excelencia.

Desde esta perspectiva, el docente de la unidad curricular Proyectos asume funciones de investigador, tutor, asesor, facilitador y mediador de aprendizajes, que lo llevan a formar a un individuo con visión crítica, creativa y reflexiva ante las demandas sociales, considerándolo un recurso o talento intelectual para el desarrollo de las capacidades científico-tecnológicos como fuente innovadora de transformación social.

Por otra parte, Delgado y Alfonzo (2019), afirman que el:

Docente-investigador implica reflexionar permanentemente, asumir una actitud crítica sobre lo aprendido en su formación y notificar saberes con sus pares y estudiantes, para establecer sinergias entre lo que se aprende e investiga, además sobre lo que se enseña y el para qué. Imparcialmente, la figura del docente-investigador lleva a considerar al sujeto como un representante reflexivo capaz de articular la praxis docente con 
la investigación (pág. 203-204).

Al respecto, el abordaje teórico que aquí presentamos forma parte de una revisión sistemática sobre el tema de estudio, rol del docente de proyectos, que orienta procesos investigativos con otros colegas docentes y estudiantes, formando parte de la construcción de conocimientos aplicables a la realidad del entorno socio-productivo.

Por último, este artículo termina con las reflexiones finales que invitan al docente a adquirir diferentes competencias, estrategias de apoyo y coordinación para promover en sus estudiantes proyectos de investigación de calidad, mejorando el proceso de enseñanza-aprendizaje en el contexto de la educación universitaria.

\section{Contexto temático y abordaje teórico}

\subsection{El rol del docente-investigador}

En Venezuela, ante el proceso de transformación universitaria que se ha venido gestando en los últimos años, exige delimitar las competencias del docente que administra unidades curriculares como Proyectos, los cuales deben desarrollar apropiadamente sus funciones y/o roles que resultan de los PNF, nuevo escenario de actuación profesional en el que intervienen los estudiantes, el docente con los demás asesores y las comunidades.

Ante esto, Fernández (2003), citado por Ruiz y Aguilar (2017a), afirman que el rol de los docentes en las universidades ha cambiado, el cual: "ha pasado de expositor a guía, a administrador de medios" (pág. 39). Asimismo, el docente trabaja bajo entornos presencial o virtual, y demanda formas de enseñanza activas, eficaces y potenciadoras del proceso de construcción del conocimiento en los estudiantes.

Por su parte, la labor del docente de proyectos se centra en un conjunto de actividades que engloban diversos roles como: investigador, facilitador, 
formador, evaluador, planificador, tutor, mediador y orientador; los cuales surgen de la experiencia educativa, del contacto directo con la realidad, la interrelación con los estudiantes y las comunidades abordadas. Al respecto, Hernández (2009a), plantea que el docente que ejerce funciones de investigación en la formación de estudiantes:

Su rol se encuentra en permanentemente construcción de saberes al interior de su actividad académica, en el aula o fuera de ella, en prácticas de indagación en diversos campos del conocimiento; espacios donde convergen una serie de significados, valores, actitudes, aptitudes y prácticas alrededor de un núcleo común: el currículo (pág. 15).

Asimismo, Hernández (2009b): afirma que el docente investigador, es un "sujeto de la educación con un ethos orientado hacia su función de educador, formador e investigador" (pág. 14); por otra parte, el docente investigador tiene la responsabilidad de producir, desarrollar y socializar el conocimiento desde su propio punto de vista y en consenso con los demás actores involucrados en el proceso de aprendizaje; precisando retroalimentación y reflexión, al mismo tiempo concibe la formación de profesionales desde el concepto integral orientado a la resolución de problemas reales (pág. 14-17).

En relación a esto, el docente investigador que administra la Unidad curricular (UC) Proyectos está involucrado directamente en la formación educativa de sus participantes, brindándole herramientas, procedimientos, estrategias de toma de decisiones y todo tipo de medios para la realización de labores investigativas en sinergia con el sector socio-productivo a abordar, lo que le permite al estudiante transferir los conocimientos adquiridos a su vida diaria con sentido socio-critico, para la producción de un bien o un servicio único vinculado a las comunidades.

Para Saby (2012a), el rol del docente investigador: "depende del modo como se asuma la docencia investigativa y la relación entre investigación y 
enseñanza" (pág. 26). En este sentido, el docente debe fomentar una cultura investigativa en sus estudiantes de acuerdo a funciones como:

- Fomentar el espíritu de la curiosidad y el descubrimiento, la búsqueda permanente por lo desconocido.

- Orientar proyectos de investigación a través del uso de metodologías adecuadas a las ciencias naturales y sociales.

- Propiciar el debate e intercambio de ideas, y fomentar el respeto a la práctica investigativa cumpliendo códigos éticos.

- Ofrecer apoyo a los estudiantes en cuanto a materiales, insumos, equipos, herramientas, laboratorios, bases de datos, redes de investigación, entre otros.

Por otra parte, dentro de las labores del docente de la UC proyectos, aparte de investigador incluye ser tutor-asesor, lo cual lo llevar a orientar al estudiante en todas las actividades académicas y de investigación que requiere para la elaboración del proyecto. Al respecto, De Del Castillo (2007), define al tutor-asesor como el:

Profesor investigador que se responsabiliza académicamente de un estudiante en la formación específica de un proyecto de investigación, teniendo una relación unipersonal, directa e individualizada de acuerdo con el plan de estudios correspondiente, así como la asesoría correspondiente durante todo el proceso de realización (pág. 23).

En este orden de ideas, en la figura 1, se destacan las principales características y tareas del docente investigador de la UC Proyectos, convirtiéndolo en tutor-asesor de las actividades que ejecutan los estudiantes durante el desarrollo de su proyecto de investigación. 
Figura 1. Actividades ejecutadas en la UC Proyectos.

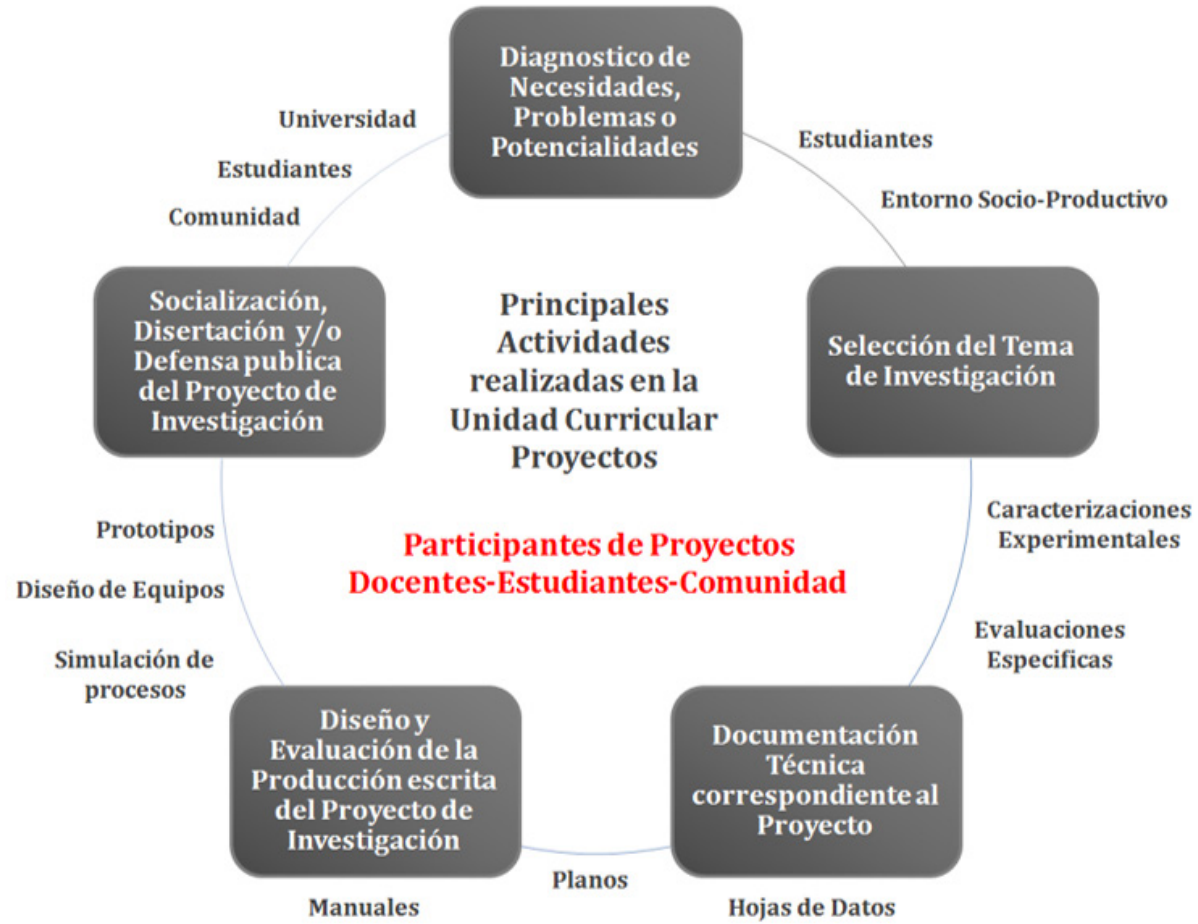

Fuente: Las Autoras (2019).

En relación a esto, se describen las siguientes tareas del docente investigador de proyectos:

a). La interacción académica en proyectos, está conformada por los estudiantes y el profesor-tutor como mediador de los aprendizajes, quienes escogerán una comunidad o entorno socio-productivo para la ejecución de proyectos durante su formación académica.

b). Las etapas del proyecto de investigación implican: el diseño y elaboración del proyecto, la investigación y producción escrita del proyecto, y la disertación o defensa pública del proyecto.

c). El tutor-asesor y los estudiantes se reúnen por lo menos una vez a la semana, durante tres o cuatro horas aproximadamente, dependiendo el PNF. Por su parte, los equipos de proyectos están conformados por dos 
o más estudiantes (máximo cinco). Los estudiantes interaccionan con tutor con el fin de que sean evaluados los resultados, alcances, y efectos de los proyectos.

d). El tutor en conjunto con los estudiantes y la comunidad escogida, realizan un diagnostico participativo para la detección de necesidades, problemas y/o potencialidades presentes en el sector.

e). Después de definir el tema de investigación, el tutor asigna diversas tareas a los estudiantes como: la realización de la documentación técnica de acuerdo a los trayectos y programas de formación, la evaluación de impactos desde el punto de vista socio-económico, ambiental y/o tecnológico. Asimismo, el tutor debe apoyar a los estudiantes ante las labores administrativas relativas a la realización de los proyectos, considerando los recursos (económicos, materiales, insumos, entre otros).

f). Dependiendo el PNF, la realización de los proyectos de investigación de los estudiantes en conjunto con el docente implica: evaluaciones técnicas, caracterizaciones experimentales de laboratorio, elaboración de manuales, hojas de datos, planos, diagramas de procesos, diseño o construcción de prototipos y unidades móviles, diseños de procesos y/o equipos ingenieriles, simulación de procesos, entre otros.

g). El docente debe facilitar y orientar a los estudiantes en lo que respecta a los procedimientos, herramientas e instrumentos necesarios para la búsqueda de información relativa a los proyectos, así como reflexionar sobre el conocimiento y la práctica; y aprovechar las tecnologías actuales y promover la innovación de estas.

h). Revisar detalladamente y críticamente los avances en el trabajo escrito del proyecto de investigación, asimismo debe corregir y comparar con versiones anteriores, para finalmente evaluar la presentación pública por parte de los estudiantes, donde participan el representante de la 
comunidad y el jurado evaluador del proyecto.

Ante esto, el docente es quien permite legitimar los conocimientos y promover el desarrollo de la ciencia a través de la investigación en los proyectos, igualmente a partir de los resultados de las labores investigativas permite la construcción colectiva de la ciencia, la transformación y el avance del conocimiento, como resultado de los descubrimientos de los trabajos de investigación que realiza en sinergia con sus estudiantes.

\subsection{Perfil del docente investigador}

El docente a partir su formación académica y los conocimientos disciplinarios adquiridos, debe propiciar un escenario acorde para el intercambio de saberes entre los distintos actores que participan en el proyecto de investigación (demás asesores, estudiantes y comunidad), permitiendo la construcción de un aprendizaje significativo e integrando los distintos programas y contenidos sinópticos que componen el pensum de estudios. En este sentido, Castro (2014a), plantea que el perfil profesional del docente en universidades tecnológicas: "abarca tres ámbitos especiales de dominio: experiencia en áreas productivas, amplio conocimiento disciplinario y disponibilidad de tiempo completo" (pág. 118). Ante esto, el perfil del docente orientado hacia su función de tutor-asesor e investigador como parte del nuevo modelo educativo establecido por Estado venezolano a través de los PNF, abarca las siguientes características:

- Se requiere que el docente tenga experiencia teórica y práctica, relacionado con su ejercicio profesional en el área educativa al que está adscrito.

- Se requiere que el docente tenga formación y/o experiencia en el área relativa a la gestión de proyectos, con el fin de desempeñar 
adecuadamente funciones academias y de investigación. Además, un poseer un amplio conocimiento multidisciplinario adecuado al nivel de formación académica y de investigación (postgrado).

Sin embargo, el perfil académico de los docentes de Proyectos se encuentra asociado a su profesión de: ingeniero en diferentes áreas como procesos químicos, mecánica, electricidad, electrónica, hidrocarburos, instrumentación, higiene y seguridad laboral, mantenimiento mecánico, materiales industriales, otras carreras como agrónomo, comunicador social, abogado, medico, economista, entre otros; los cuales no han sido formados como profesores, asumiendo este rol docente sin la debida experiencia, en algunos casos no poseen estrategias para el manejo disciplina que enseña y no ha sido formado en investigación.

Por otra parte, los docentes ante el proceso de transformación universitaria, sobre todo los docentes más veteranos han tenido que adaptarse a las nuevas políticas educativas, trayendo consecuencia respuestas negativas, como resistencia a los cambios y paradigmas actuales, en algunos casos no tienen dominio sobre los temas específicos relativos a proyectos. Así como también, los docentes suelen ser reacios y asumen posturas reactivas a la hora de asumir nuevas funciones, entre ellas la de investigación. Es motivo de reflexión para estas universidades, que se ejecuten programas de actualización continua para los docentes de Proyectos, como apoyo al desarrollo de destrezas ante la práctica científica, y enriquecimiento de sus actividades de investigación, y orientada a mejorar la práctica profesional del docente.

Por otra parte, el docente de proyectos se involucra constantemente con investigaciones en diversas disciplinas o áreas del saber, lo que lo hace un investigador nato y apasionado por esta actividad. Por lo tanto, quienes asumen esta responsabilidad de llevar estas unidades curriculares, deben 
tener vocación para ello, asumen el rol de ser útil y de ayudar a otras personas, a través de la ejecución de proyectos de investigación aplicables a las comunidades.

Asimismo, dentro de la UC Proyectos existe una diversidad de saberes que en algunos casos el docente-tutor no lo sabe todo, lo que lo convierte en investigador de su propio conocimiento complementando, experimentando y descubriendo en conjunto con los estudiantes, convirtiéndose en coautor de los proyectos de investigación.

\subsection{Competencias del docente investigador}

En la actualidad el docente debe desarrollar un conjunto habilidades, aptitudes, actitudes, conocimientos, destrezas y valores necesarios para llevar a cabo su función como investigador y tutor-asesor de investigaciones aplicables en entornos socio-educativos y socio-productivos. Al respecto, Ruiz y Aguilar (2017b): distinguen claramente dos tipos de competencias que debe tener el docente universitario: competencias transversales constituidas por valores, actitudes, destrezas y cualidades personales que acompañan la labor docente; y competencias específicas relativas a las funciones del profesor universitario, docencia, investigación, y extensión (pág. 42).

En este orden de ideas, López y Pérez (2018), afirman que el docente: "debe contar con un conjunto de competencias tutoriales que le permitan satisfacer las demandas planteadas" (pág. 50). En relación a esto, ser un buen profesor-asesor de proyectos implica poseer un conjunto de competencias que le permita abordar situaciones del entorno, que lo llevan a resolver problemas dentro de un contexto educativo universitario.

Las autoras consideran las siguientes competencias mostradas en la figura 2, las cuales se deben adquirir por el profesor-asesor de la UC proyectos, y de esta manera cumplir su rol de docente investigador ante la practica social de la ciencia, apoyado en los postulados de Álvarez y Villareal 
(2004a); Barrón (2009a); Cano (2009a); y Saby (2012b). A saber, se tienen:

Figura 2. Competencias del Docente de la UC Proyectos.

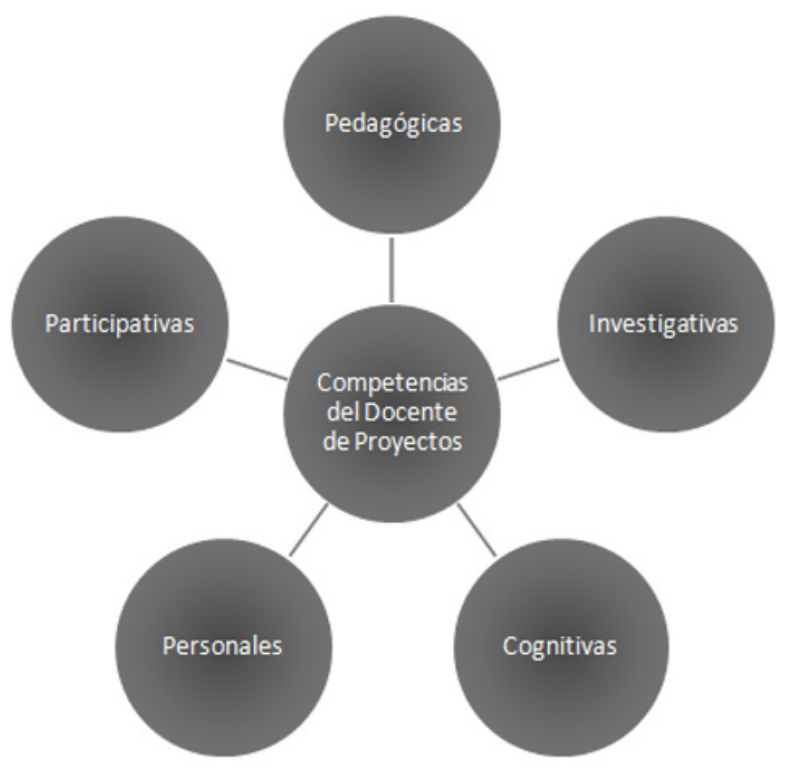

Fuente: Las Autoras (2019).

Competencias pedagógicas: estas competencias son facilitadoras de los procesos de aprendizaje, corresponde a las estrategias de intervención didácticas que permiten evaluar de manera efectiva y creativa los procesos de enseñanza-aprendizaje en los estudiantes. Dichas competencias son de carácter práctico, manifestado a través de la capacidad crítica para integrar la teoría con la práctica y para intervenir eficazmente la realidad con el entorno socio-educativo y socio-productivo. El docente debe poseer una adecuada capacidad de planificación y organización de los contenidos y programas, gestión de recursos de aprendizaje, de aprendizaje y de evaluación.

Competencias investigativas: el docente debe tener interés por crear, descubrir e innovar el conocimiento; son las características propias que lo definen como investigador en el proceso de elaboración de proyectos, estas competencias son el motor promotor de la práctica científica, igualmente el 
docente debe dominar conceptos de la ciencia, tecnología e innovación, despertar su compromiso para generar y desarrollar ideas con sentido crítico, interpretando el entorno y aprovechar oportunidades, y por supuesto, enseñar a otros a hacerlo, crear condiciones para orientar la curiosidad y el descubrimiento a lo desconocido. El docente debe proponer soluciones a problemas encontrados, y comprender el beneficio de la investigación para dar respuesta a los problemas del entorno.

Competencias cognitivas: relacionadas con el ámbito profesional del docente, lo que le permiten aplicar y dominar conocimientos especializados en las tareas de investigación. A partir de estas competencias, el docente ejerce acciones concretas, procedimientos y métodos para difundir el conocimiento útil para el aprendizaje de los demás y para la toma de decisiones. También, el docente debe concebir su profesión como un trabajo de constante aprendizaje, ya que la formación académica es una competencia que se le exige al mismo en esta sociedad que se prepara cada día ante los cambios mundiales.

Competencias personales: de refieren a las habilidades o destrezas de carácter personalizado; relacionadas con saber actuar en concordancia con los propios principios e ideas; saber tratar con distintos colectivos y situaciones que se le presenten en las labores de investigación; asumir las responsabilidades que le correspondan en cada situación; saber tomar decisiones válidas y pertinentes; saber hacer frente a las frustraciones e inconvenientes que se le presenten; tener una actitud abierta al cambio, a la empatía, respetar los códigos o principios éticos y científicos.

Competencias participativas: relacionas a la práctica social, es la habilidad que tiene el docente para estar siempre dispuesto al diálogo, al entendimiento y la comprensión con sus actores socio-educativos (estudiantes, profesores, comunidad, entre otros). El docente debe desarrollar habilidades de comunicación, crear un clima para participación, análisis y 
discusión sobre los diferentes temas abordados en los proyectos de investigación. Asimismo, proveer un feedback o retroalimentación de los conocimientos adquiridos con el fin de mejorar la investigación y la comunicación en los proyectos.

En resumen, el docente de proyectos debe adquirir una serie de competencias cognitivas, académicas, destrezas sociales, personales, y sociales que le permiten asumir con un buen desempeño el rol de docente investigador, supervisor y asesor de las investigaciones llevadas a cabo por sus estudiantes. Dichas competencias se construyen en el día a día del profesor de la UC Proyectos, de la experiencia con el trabajo de investigación asumido con sus estudiantes que lo diferenciaran del resto de los docentes de las diferentes unidades curriculares, y responden a las demandas de los estudiantes, así como también se deben analizar y potenciar las practicas adquiridas, e incorporar aquellas competencias necesarias para llevar a cabo de manera exitosa su labor docente y de investigación.

\subsection{Dificultades encontradas durante las prácticas del docente de la UC Proyectos}

Durante los procesos de asesoría de proyectos, se pueden presentar ciertos problemas que pueden poner el riesgo la ejecución del trabajo, afectando la calidad del proyecto de investigación. Las autoras consideran algunas dificultades y errores que se tienen a la hora de asumir el rol del tutorasesor de trabajos de investigación; apoyado en los postulados de Morillo (2009a): sostiene que La falta de fuentes de información, pueden dificultar la elaboración de proyectos de investigación, para lo cual el docente investigador a partir de su conocimiento y experiencia, debe enseñar a los estudiantes el uso de métodos, procedimientos y técnicas para la búsqueda de fuentes de información documentales y de campo (pág. 925).

De igual forma, Morillo (2009b): expone que La falta de preparación 
del docente, de proyectos sobre áreas específicas, la falta de la práctica de investigación y escasa aplicación de herramientas metodológicas y de estrategias para abordar a las comunidades, suelen incidir significativamente en los estudiantes, provocando apatía o desinterés por lo que se investiga (pág. 925).

De acuerdo con Morillo (2009c): La falta de autonomía o libertad intelectual hacia el estudiante, en ocasiones el docente interviene en el desarrollo de temas de interés para él, coartando la creatividad e iniciativa del estudiante, considerando que a menudo los tutores tienen gran poder de convencimiento o influencia en sus estudiantes (pág. 927).

Por otro lado, debido a la migración del talento humano estas universidades carecen de personal técnico calificado, conllevando a que se aumente el número de secciones de proyectos al personal activo, por lo que el docente dedica menos tiempo a los grupos de trabajo, inclusive aquellos docentes que cumplen funciones administrativas y dedican menos tiempo a proyectos, retardan la lectura de manuscritos presentados por los estudiantes, e incumpliendo su rol de docente-asesor-tutor de proyectos.

Todas estas deficiencias en los procesos de asesoría de proyectos provocan desmotivación en los estudiantes y en muchos casos, se refleja en la calidad de la investigación, llena de carencias y que son mostradas en la defensa pública del proyecto. Es importante que los docentes reconozcan éticamente cuando no estén capacitados para asumir tareas específicas del trabajo de investigación, y que requieren de la ayuda de un asesor en áreas distintas a las de su competencia, tanto el docente de proyectos como los demás asesores deben trabajar en equipo, al mismo tiempo deben considerar las opiniones y sugerencias emitidas por especialistas sobre las limitaciones que se presentan a lo largo del desarrollo de la investigación. 


\section{Metodología}

La metodología utilizada fue la revisión bibliográfica, consultándose trabajos de investigaciones y artículos de revistas especializadas relacionados con el tema de estudio "Rol del docente investigador", lo cual representó la base teórica de la investigación.

La revisión bibliográfica involucró un periodo de tiempo donde se reflexionó sobre los postulados de Hernández (2009c); Saby (2012c); Castro (2014b); Álvarez y Villareal (2004b); Barrón (2009b); Cano (2009b); y Morillo (2009d); entre otros. Por su parte, esta revisión bibliográfica permitió reflexionar sobre el rol del docente investigador ante la práctica social de la ciencia, con el fin de que el mismo alcance ciertas competencias para cumplir adecuadamente su rol como formador de investigadores en temas de interés científico.

\section{Reflexiones finales}

El docente de proyectos es que aquel que orienta a los estudiantes a investigar, el docente investigador propiamente es aquel que investiga y enseña a investigar, por lo que los proyectos se convierten en espacios de formación con orientación científica-tecnológica, donde se integran una multiplicidad de saberes para dar respuesta a un entorno. Las universidades deben destinar recursos para mejorar programas de formación docente antes las exigencias que tiene que asumir el profesor que administra la unidad curricular Proyectos, con el fin de garantizar las condiciones necesarias para el óptimo desempeño de la docencia, investigación y extensión.

Esta formación permitirá a los docentes desarrollar, adquirir y fortalecer competencias necesarias para llevar a cabo con éxito su labor como investigador y asesor de proyectos. También, el docente debe adaptarse a la realidad laboral actual utilizando distintos modos de formación (presencia, semipresencial y/o distancia), y utilizar recursos tecnológicos para fortaleces 
las labores investigativas (videoconferencias, tutorías online, entre otros).

Además, la investigación se considera un elemento integrador del proceso de enseñanza-aprendizaje y a partir de los proyectos, el docente investigador maneja una práctica didáctica y social, a partir de la solución de problemas o situaciones reales que se dan en las comunidades, todo esto tomando en consideración su rol como formador de un estudiante crítico con valores éticos, morales, culturales.

Estos proyectos requieren un constante seguimiento por parte de docente investigador, lo cuales lo convierte en gestor de sus propias actividades de forma independiente, participando en la planificación de tareas en conjunto los estudiantes y las comunidades. El docente debe diseñar estrategias para recoger la información requerida para la investigación, con la finalidad de identificar desde el primer momento las necesidades de las comunidades, ya que a partir del diagnóstico se establece en gran medida el éxito del proyecto.

A manera de conclusión, se puede decir que todos los tópicos abordados en el presente artículo, específicamente los problemas que se tienen a la hora de elaborar proyectos de investigación, deben apuntar al fortalecimiento de las competencias docentes, conllevando al mejorando de la calidad en el trabajo de investigación y, paralelamente, del proceso de enseñanza-aprendizaje.

\section{Referencias}

Álvarez, C., \& Villareal, Z. (2004a,b). Modelo de competencias requeridas para desempeñar el rol del tutor en una institución de Educación Superior. Tesis presentada en el cumplimiento parcial de los requerimientos para obtener el grado de Maestría en Ciencias de la Comunicación. México: Universidad Autónoma de Nuevo León. Recuperado de: http://eprints.uanl.mx/1622/1/1020146143.PDF 
Barrón, M. (2009a,b). Docencia universitaria y competencias didácticas. Perfiles Educativos, XXXI(125), 76-87, e-ISSN: 0185-2698. Recuperado de: http://www.redalyc.org/articulo.oa?id=13211980006

Castro, R. (2014a,b). Análisis de las buenas prácticas del tutor universitario: estudio caso en la Universidad Tecnológica de Chihuahua de México. Tesis Doctoral. Bellaterra, España: Universidad Autónoma de Barcelona. Recuperado de:

https://ddd.uab.cat/pub/tesis/2014/hdl 10803 133264/racc1de1.pdf

Cano, R. (2009a,b) Tutoría universitaria y aprendizaje por competencias ¿Cómo lograrlo?. Revista Electrónica Interuniversitaria de Formación del Profesorado, 12(1), 181-204, eISSN: 1575-0965. Recuperado de:

http://www.redalyc.org/articulo.oa?id=217015332014

De Del Castillo, J. (2007). Conversando con tutores y asesores de tesis. Visión Gerencial, 6(Número Especial), 16-32, e-ISSN: 1317-8822. Recuperado de:

http://www.saber.ula.ve/bitstream/handle/123456789/25157/articulo2.p df;jsessionid=EA421D8B574851DC86904A6A419B27C0?sequence $=2$

Delgado, Y., \& Alfonzo, R. (2019). Competencias Investigativas del Docente Construidas durante la Formación Universitaria. Revista Scientific, 4(13), 200-220, e-ISSN: 2542-2987. Recuperado de:

https://doi.org/10.29394/Scientific.issn.2542-2987.2019.4.13.10.200$\underline{220}$

Hernández, I. (2009a,b,c). El docente investigador en la formación de profesionales. Revista Virtual Universidad Católica del Norte, (27), 121, e-ISSN: 0124-5821. Recuperado de: http://www.redalyc.org/articulo.oa?id $=194215432011$ López, C., \& Pérez, M. (2018). Competencias Tutoriales en los Programas 
de Postgrado: Una Mirada desde la Experiencia Venezolana. Revista Scientific, 3(9), 39-60, e-ISSN: 2542-2987. Recuperado de: https://doi.org/10.29394/Scientific.issn.2542-2987.2018.3.9.2.39-60

Morillo, M. (2009a,b,c,d). Labor del tutor y asesor de trabajo de investigación. Experiencias e incentivos. Educere, 13(47), 919-930, e-ISSN: 1316-4910. Recuperado de:

http://www.redalyc.org/articulo.oa?id=35616673004

Rodríguez, J. (2016). Redimensión del proceso de aprendizaje para la transformación de los Institutos y Colegios Universitarios en Venezuela. Tesis presentada como Requisito de Mérito ante la Universidad de Carabobo para optar al Título de Doctor en Educación. Venezuela: Universidad de Carabobo. Recuperado de:

http://mriuc.bc.uc.edu.ve/bitstream/handle/123456789/4116/jrodriguez. pdf?sequence $=1$

Ruiz, M., \& Aguilar, R. (2017). Competencias del profesor universitario: elaboración y validación de un cuestionario de autoevaluación. Revista Iberoamericana de Educación Superior (RIES), VIII(21), 37-65, e-ISSN: 2007-2872. Recuperado de:

https://www.redalyc.org/articulo.oa?id=299149615003

Saby, J. (2012a,b,c). El rol del docente-investigador en el marco de la investigación formativa. Revista PAPELES, 4(8), 23-30, ISSN: 01230670; e-ISSN: 2346-0911. Recuperado de:

http://revistas.uan.edu.co/index.php/papeles/article/view/225 


\section{Eddymar María Flores Nessi}

e-mail: eddymarf.nessi@gmail.com

Nacida en la ciudad de Cabimas, Estado Zulia, Venezuela,

el 11 de marzo del año 1984. Ingeniera Química, Universidad del Zulia (LUZ, 2007); Magister en Gerencia de Proyectos de Investigación y Desarrollo, Universidad Dr. Rafael Belloso Chacín (URBE, 2016); Con experiencia docente a nivel universitario desde hace 10 años. Actualmente Docente a Tiempo Completo en la Universidad Politécnica Territorial del Zulia (UPTZ); Docente de las Unidades Curriculares: Proyectos, Ingeniería de las Reacciones Químicas, Ingeniera Ambiental; Tutora y jurado evaluador de trabajos y proyectos de Investigación; Experiencia en la asesoría y consultoría ambiental referida a la elaboración de estudios de impacto ambiental, diagnósticos ambientales y tramitación de autorizaciones ambientales. 


\section{Alba Carolina Loaiza Falcón}

e-mail: albaloaiza.104@gmail.com

Nacida en la ciudad de Cabimas, estado Zulia, Venezuela,

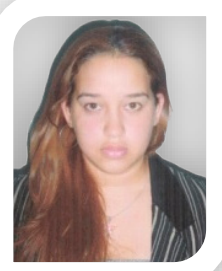

el 6 de febrero del año 1985. Ingeniera Mecánica, Universidad del Zulia (LUZ, 2007); Magister en Ciencia y

Tecnología de los Materiales, Universidad de la Habana (2015); Magister en Gerencia de Empresas Mención: Operaciones, Universidad del Zulia (LUZ, 2016); con experiencia docente a nivel universitario desde hace 11 años. Actualmente, docente en la categoría de Agregado a Tiempo Completo en la Universidad Politécnica Territorial del Zulia (UPTZ); Docente de las unidades curriculares: Algebra, Calculo, Matemática, Física, Laboratorio de física; Tutora y jurado evaluador de trabajos y proyectos de investigación, experiencia en departamento de división docente, planificación de cursos de verano, planificación académica y estudios de mercado; también experiencia laboral en departamentos de crédito y cobranza, auditorias de campo. 


\section{Gisemar Ninoska Rojas de Ricardo \\ e-mail: gisemarninoska@gmail.com}

Nacida en la ciudad de Cabimas, Estado Zulia,

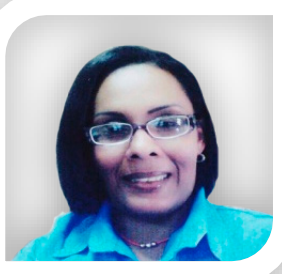

Venezuela, el 13 de febrero del año 1974. Licenciada en Educación Integral Mención Castellano, Literatura y Ciencias Biológicas de la Universidad Nacional Experimental "Rafael María Baralt" (UNERMB, 2000); Magíster Scientiarum en Administración de la Educación Básica de la mencionada casa de estudios (UNERMB, 2005); Docente con Función Directiva en la Escuela Básica Nacional "Don Rómulo Gallegos" desde febrero del año 2017. 